\title{
The Novel Liquid Crystalline Organic Semiconducting Oligomers Incorporating $N$ - Heterocyclic Carbazole Moieties for fluorescent OLEDs
}

\author{
Guang Hu${ }^{a}$, Stuart P. Kitney ${ }^{b}$, Stephen M. Kelly ${ }^{a}$ \\ William Harrison $^{b}$, Mary $\mathrm{O}^{\prime} \mathrm{Neill}^{a}$ \\ ${ }^{a}$ School of Mathematics and Physical Sciences, University of Hull, Cottingham Road, Hull, HU6 \\ $7 R X, U K$ \\ ${ }^{b}$ Polar OLED, University of Hull, Cottingham Road, Hull, HU6 7RX, UK
}

\begin{abstract}
A novel class of nematic liquid crystalline organic semiconducting oligomers incorporating $\mathrm{N}$ heterocyclic carbazole moieties has been synthesised using simple and highly efficient reaction pathways. The electroluminescent colour of these novel oligomers can be varied in a controlled manner by molecular design. The values of the ionization potential and the electron affinity of these electroluminescent oligomers can also be matched by structural design to the HOMO energy level of the electron-blocking layer and the LUMO energy level of electron-transporting layer in the OLEDs to create low charge-injection barriers for electrons and holes, respectively leading to electroluminescence with an efficacy up to $4.1 \mathrm{~cd} \mathrm{~A}^{-1}$.
\end{abstract}

Keywords: Nematic Liquid Crystalline, Oligomers, Carbazole, Synthesis, Electroluminescent, OLED

\section{Introduction}

Organic light emitting diodes (OLEDs) with high information content, active matrix addressing and full colour are a type of novel flat-panel display, which does not require backlighting and other mostly light-absorbing - components, such as the diffusers, colour filters and polarisers, used in LCDs [1-5]. Consequently, OLEDs are more efficient in terms of light emission due to the absence of so many light-absorbing or scattering layers and because no backlight is required. Particularly, multi-stack OLEDs with additional carrier transporting or blocking layers are introduced between 
the hole-/electron-transporting layer and the anode of tri-layer OLEDs, for example, to produce a lower barrier for hole injection into the other layers of the device. Many variants of multi-stack OLEDs have been developed with improved overall performance, e.g., in terms of brightness, power conversion efficiency, threshold voltage and operating voltage, etc. [6-9]

OLEDs require organic charge-transporting and electroluminescent materials. One of the advantages of using organic materials for electroluminescence is that they can be designed specifically with the desired functions, such as high hole or electron mobility and efficient electroluminescence. Furthermore, the electron affinity (EA, LUMO energy level) and ionisation potentials (IP, HOMO energy level) of charge-transport materials can be tuned to better match the work functions of electrodes [10-12]. These requirements for the material properties can be achieved by the design and synthesis of a variety of organic molecules. Specifically, compounds with a high concentration of highly conjugated aromatic rings, such as 1,4-disubstituted phenylene or 2,5disubstituted thiophene rings, in the molecular core, can form conjugated electron clouds with a high degree of delocalization, which renders aromatic organic compounds semiconducting under certain conditions [13-15]. Currently, the materials for OLEDs can be usually classified as lowmolecular-mass materials (LMMMs) [16-20], polymers [21-29] and oligomers [30-36].

Liquid crystalline organic semiconductors [37-43] have found use in plastic electronic devices due to the tendency of such organic semiconductors in the fluid, self-assembling nematic phase - with an intrinsically low viscosity - to form uniform, thin layers or films, with a low concentration of charge traps, on device substrate surfaces. Deposition from solution from common organic solvents can be achieved using, for example, doctor blade, drop casting or spin coating techniques. The presence of a glassy nematic state above room temperature for oligomers means that there is no need to photochemically crosslink oligomeric liquid crystalline organic semiconductors in order to stabilize the fluid film on the device substrate.

The presence of the hetero-atoms in aromatic heterocyclic moieties in some highly conjugated organic semiconductors contributes to the improved optoelectronic properties of such materials. For example, the carbazole moiety has long been recognized as an excellent construction of 
photoconductive organic semiconductors due to strong fluorescence, good hole-transporting ability, low redox potential and chemical/environmental stability [44-51]. Compared with the corresponding 2,7-disubstituted fluorene moiety, the electron-donating nature of the nitrogen hetero-atoms present in the carbazole moieties can increase the electron density of the delocalized $\pi$-electronic cloud and thereby affect the magnitude of the physical properties of interest for OLEDs.

\section{Experimental}

\subsection{Materials and Methods}

All commercially available starting materials and reaction intermediates, reagents and solvents were obtained from Aldrich, Strem Chem. Inc., Acros or Lancaster Synthesis and were used as supplied, unless otherwise stated. Tetrahydrofuran (THF) was pre-dried using 3A molecular sieves, which had been dried at $250^{\circ} \mathrm{C}$ to ensure optimum activity. All reactions were carried out using a dry atmosphere of nitrogen unless water was present as solvent or reagent, and the temperatures were measured internally. Mass spectra were recorded for each structure. Compounds with a molecular mass less than $800 \mathrm{~g} / \mathrm{mol}$ are analysed using a gas chromatography/mass spectrometer (GC/MS)Quadrupole MS/ Perkin Elmer autosystem XL GC with Electron Impact (EI) at a source temperature of $200^{\circ} \mathrm{C}$. Structures with a higher molecular mass are determined using a Maldi-MS Bruker autoflex speed using a 384 spot anchorchip 800 as a target. Samples are dissolved in DCM with HABA (2-(4-hydroxyphenylazo)benzoic acid) matrix (1:10 respectively). $\mathrm{M}^{+}$is identified as the mass ion of the material. Infrared (IR) spectra were recorded using a PerkinElmer Paragon 1000 Fourier Transform-Infrared (FT-IR) spectrometer and H and C NMR spectra were recorded using a JEOL Lambda 400 spectrometer with an internal standard of tetramethylsilane (TMS). Thin layer chromatography (TLC) using aluminium-backed TLC plates coated with silica gel (60 F254 Merck) was used to measure every reaction progress. The purification of reaction inter-mediates and final products was carried out by gravity column chromatography, using silica gel (40-63 microns,60 A) obtained from Fluorochem. The purity and chemical composition of the final products was determined by elemental analysis (C, H, N and S) using a Fisons EA 1108 CHN. The melting point and transition temperatures for each final compound were measured using polarising optical microscopy (POM), with a Mettler FP-5 hot-stage and an Olympus BH2 polarising microscope. 
Differential Scanning Calorimetry (DSC) was also used consisting of a Mettler Toledo DSC1, to determine the enthalpy of transitions and transition temperatures at a heating rate of $10^{\circ} \mathrm{C}$ per minute. Good correlation between POM and DSC transition temperatures was noted, within $1^{\circ} \mathrm{C}$. UVVisible absorbance spectra were measured using a Thermo Scientific Evolution 220 UV-Visible Spectrometer. The compounds were dissolved in chloroform, placed in a quartz cuvette and a spectrum recorded from $750 \mathrm{~nm}$ to $190 \mathrm{~nm}$ using a bandwidth of $2 \mathrm{~nm}$. The ionisation potential (IP) of the compounds were measured electrochemically by cyclic voltammetry using a computercontrolled scanning potentiostat (Solartron 1285), which functions as wave generator, potentiostat and current-to-voltage converter. The Corrware and Corrview software packages were used to control and record the cyclic voltammetry experiments, respectively. $1 \mathrm{mM}$ of the compound was dissolved in $5 \mathrm{~cm}^{3}$ of an electrolytic solution of $0.3 \mathrm{M}$ tetrabutylammonium hexafluorophosphate (TBAHFP 6) in DCM. The solution was placed in a standard three-electrode electrochemical cell. A glassy carbon electrode was used as the working electrode. Silver/silver chloride (3 M NaCl and saturated $\mathrm{Ag} / \mathrm{Cl}$ ) and a platinum wire were used as the reference and counter electrodes, respectively. The electrolyte was recrystallised twice before use. Oxygen contamination was avoided by purging the solution with dry argon before each measurement. The measured potentials were corrected to an internal ferrocene reference added at the end of each measurement. A typical scan rate of $20 \mathrm{mV} \mathrm{s}$ -

${ }^{1}$ was used, and two scans were performed to check the repeatability. The onset potential for oxidation, Eox, is clearly defined by a step change in current and is obtained from the intersection of the two tangents at the current discontinuity based on the empirical relationship proposed by Bredas, IP 1/4 [Eox p 4.4] eV. We were unable to measure a value for the reduction potential because of the limited working range of the electrolyte. However, the electron affinity (EA) was estimated by subtraction of the optical band edge (Eg), taken as the energy of the onset of absorption of the compound from the IP. Although this approximation does not include a correction for the exciton binding energy, the values obtained agree within $\pm 0.05 \mathrm{eV}$ with those measured electrochemically in our laboratory for other classes of reactive mesogen.

\subsection{OLED Fabrication}

A general schematic representation of the configuration of the OLEDs fabricated is shown in Figure 1. A layer of poly(3,4-ethylenedioxythiophene):polystyrenesulfonate (PEDOT:PSS Sigma-Aldrich), used as the hole-injection layer due to its high conductivity (ca $3.0 \times 10^{-3} \mathrm{~S} / \mathrm{cm}$ ) [52-53], was 
deposited as an hole-injection layer (30 nm) from aqueous solution on a glass substrate (Osilla) with a $100 \mathrm{~nm}$ pre-coated transparent indium tin oxide (ITO) anode by spin coating (2000 rpm for 30s) using Laurell WS-400-6NPP-Lite Spin Processer. The film thickness was measured using Dektak. N4,N4'-bis(4-\{6-[(3-Ethyloxetan-3-yl)methoxy]hexyl\}phenyl)-N4,N4'-diphenylbiphenyl-4,4'diamine (OTPD) (Luminescence Technology Corp), used as a hole-transporting layer after photochemical crosslinking [54], was deposited from a $0.5 \mathrm{wt} \%$ in toluene solution by spin-coating (2000 rpm for 30s) to form a hole-transporting layer (15 nm) on top of the PEDOT/PSS holeinjection layer. $0.5 \mathrm{wt} \%$ (4-octyloxyphenyl) phenyliodonium hexafluoroantimonate (OPPI) was added as a photoinitiator and the OTPD was photochemically crosslinked using UV irradiation with a fluend of $100 \mathrm{~mJ} \mathrm{~cm}^{-2}$. The sample was annealed at $110{ }^{\circ} \mathrm{C}$ for $5 \mathrm{~min}$. A thin layer (20 nm) of one of the compounds 1-5 was deposited as the emissive layer by spincoating at $2000 \mathrm{rpm}$ for $30 \mathrm{~s}$ from solution (1 wt\% in toluene). A layer (20 nm) of 2,7-bis(diphenylphosphoryl)-9,9'-spirobifluorene (SPPO13) used as electron-transporting layer was deposited by thermal evaporation at a pressure of $8 \times 10^{-6}$ mbar. A layer $(1 \mathrm{~nm})$ of lithium fluoride, used as the electron-injection layer was then evaporated [55], followed by an aluminium (100 nm) cathode.

\subsection{Material Synthesis}

The compounds 1-5 were synthesized as shown in reaction Scheme $\mathbf{1}$.

\section{1-Iodo-4-(octyloxy)benzene (11)}

A mixture of 4-iodophenol (20.00 g, $91.0 \mathrm{mmol})$, 1-bromooctane (16.00 g, $82.8 \mathrm{mmol}), \mathrm{K}_{2} \mathrm{CO}_{3}$ (34.40 g, $248.4 \mathrm{mmol})$ and DMF $\left(200 \mathrm{~cm}^{3}\right)$ was heated overnight at $120{ }^{\circ} \mathrm{C}$. The cooled reaction mixture was added to aqueous $\mathrm{NaOH}(91.0 \mathrm{mmol}, 1 \mathrm{~mol} / \mathrm{L})$, stirred for $1 \mathrm{~h}$ and then extracted with DCM $\left(3 \times 100 \mathrm{~cm}^{3}\right)$. The combined organic layers were washed with water $\left(2 \times 100 \mathrm{~cm}^{3}\right)$, dried $\left(\mathrm{MgSO}_{4}\right)$, filtered and then concentrated down under reduced pressure. Purification was carried out using column chromatography [silica, DCM/hexane 1:10] to afford the desired product as a colourless liquid (26.20 g, $95 \%)$.

${ }^{1} \mathrm{H}$ NMR $\left(\mathrm{CDCl}_{3}\right) \delta_{\mathrm{H}}: 7.56(2 \mathrm{H}, \mathrm{d}, J=6.8 \mathrm{~Hz}), 6.68(2 \mathrm{H}, \mathrm{d}, J=6.8 \mathrm{~Hz}), 3.91(2 \mathrm{H}, \mathrm{t}, J=6.4 \mathrm{~Hz})$, $1.79(2 \mathrm{H}, \mathrm{m}), 1.27-1.50(10 \mathrm{H}, \mathrm{m}), 0.90(3 \mathrm{H}, \mathrm{t}, J=6.8 \mathrm{~Hz})$.

MS m/z (EI): 333, $332\left(\mathrm{M}^{+}\right)$. 


\section{4-Bromo-4'-octyloxy-2-nitrobiphenyl (13)}

Copper powder (10.20 g, $160.2 \mathrm{mmol}$ ) was added over $1 \mathrm{~h}$ to a stirred, molten mixture of 2,5dibromonitrobenzene (15.00 g, $53.4 \mathrm{mmol}$ ) and 1-iodo-4-(octyloxy)benzene (21.3 g, $64.08 \mathrm{mmol}$ ) maintained at $175{ }^{\circ} \mathrm{C}$. The resultant reaction mixture was then heated at $175{ }^{\circ} \mathrm{C}$ overnight. The cooled reaction mixture was extracted into hot toluene $\left(3 \times 150 \mathrm{~cm}^{3}\right)$ and the combined organic layers filtered through silica gel. The filtrate was washed with water $\left(2 \times 200 \mathrm{~cm}^{3}\right)$, dried $\left(\mathrm{MgSO}_{4}\right)$, filtered and then evaporated under reduced pressure. Purification of the crude product was carried out using column chromatography [silica, DCM/hexane 3:10] to afford the desired product as a yellow liquid (15.10 g, $70 \%)$.

${ }^{1} \mathrm{H}$ NMR $\left(\mathrm{CDCl}_{3}\right) \delta_{\mathrm{H}}: 7.94(1 \mathrm{H}, \mathrm{d}, J=2.0 \mathrm{~Hz}), 7.69(1 \mathrm{H}, \mathrm{dd}, J=8.0 \& 1.2 \mathrm{~Hz}), 7.31(1 \mathrm{H}, \mathrm{d}, J=$ $8.4 \mathrm{~Hz}), 7.19(2 \mathrm{H}, \mathrm{d}, J=8.4 \mathrm{~Hz}), 6.94(2 \mathrm{H}, \mathrm{d}, J=8.0 \mathrm{~Hz}), 3.99(2 \mathrm{H}, \mathrm{t}, J=6.8 \mathrm{~Hz}), 1.83(2 \mathrm{H}$, quint, $J=6.8 \mathrm{~Hz}), 1.20-1.52(10 \mathrm{H}, \mathrm{m}), 0.90(3 \mathrm{H}, \mathrm{t}, J=6.8 \mathrm{~Hz})$.

MS m/z (EI): 407, $405\left(\mathrm{M}^{+}\right)$, 375, 296.

\section{2-Bromo-7-octyloxycarbazole (14)}

A mixture of 4-bromo-4'-octyloxy-2-nitrobiphenyl (10.00 g, $24.61 \mathrm{mmol})$ and triethyl phophite (20.45 g, $123.06 \mathrm{mmol}$ ) was stirred and heated at reflux overnight. The volatiles were removed under reduced pressure. The residue was then poured onto methanol $\left(300 \mathrm{~cm}^{3}\right)$ and stirred for $3 \mathrm{~h}$. The mixture was then filtered off and the precipitate was collected to afford the desired product as a white solid (7.2 g, $78 \%)$.

Melting Point $/{ }^{\circ} \mathrm{C}: 265-266$.

${ }^{1} \mathrm{H}$ NMR $\left(\mathrm{CDCl}_{3}\right) \delta_{\mathrm{H}}: 7.94(1 \mathrm{H}, \mathrm{s}), 7.89(1 \mathrm{H}, \mathrm{d}, J=8.8 \mathrm{~Hz}), 7.81(1 \mathrm{H}, \mathrm{d}, J=8.4 \mathrm{~Hz}), 7.52(1 \mathrm{H}, \mathrm{d}$, $J=1.6 \mathrm{~Hz}), 7.31(1 \mathrm{H}, \mathrm{dd}, J=8.4 \& 1.6 \mathrm{~Hz}), 6.89(1 \mathrm{H}, \mathrm{d}, J=2.2 \mathrm{~Hz}), 6.87(1 \mathrm{H}, \mathrm{dd}, J=8.8 \& 2.4$ Hz), 4.05 (2H, t, $J=6.8 \mathrm{~Hz}), 1.85$ (2H, quint, $J=6.8 \mathrm{~Hz}), 1.29-1.52(10 \mathrm{H}, \mathrm{m}), 0.90$ (3H, t, $J=6.8$ $\mathrm{Hz})$.

MS m/z (EI): 375, $373\left(\mathrm{M}^{+}\right)$.

\section{2-Bromo- $N$-octyl-7-(octyloxy)-carbazole (15)}

Sodium hydride $(\mathrm{NaH})(60 \%$ in mineral oil) $(2.14 \mathrm{~g}, 53.43 \mathrm{mmol})$ was added in a small portion into 
a solution of 2-bromo-7-octyloxycarbazole (10.00 g, $26.72 \mathrm{mmol})$ in DMF (100 mL). The resultant mixture was stirred for $1 \mathrm{~h}$ at room temperature, then heated and maintained at $50{ }^{\circ} \mathrm{C}$ for another 1 h. 1-Bromooctane (5.68 g, $29.39 \mathrm{mmol}$ ) was added and the resultant mixture stirred at $50{ }^{\circ} \mathrm{C}$ for 3 $\mathrm{h}$ and then at room temperature overnight. The reaction mixture was washed with water $\left(100 \mathrm{~cm}^{3}\right)$, and extracted with DCM $\left(3 \times 100 \mathrm{~cm}^{3}\right)$. The combined organic layers were washed with water $(2 \times$ $\left.100 \mathrm{~cm}^{3}\right)$, dried over magnesium sulfate $\left(\mathrm{MgSO}_{4}\right)$, filtered and then concentrated down under reduced pressure. The crude product was purified using column chromatography [DCM/Hexane 1:10] to afford the desired product as a white solid (20.74 g, $99 \%)$.

Melting Point $/{ }^{\circ} \mathrm{C}: 53-54$.

${ }^{1} \mathrm{H}$ NMR $\left(\mathrm{CDCl}_{3}\right) \delta_{\mathrm{H}}: 7.90(1 \mathrm{H}, \mathrm{d}, J=8.8 \mathrm{~Hz}), 7.82(1 \mathrm{H}, \mathrm{d}, J=8.4 \mathrm{~Hz}), 7.47(1 \mathrm{H}, \mathrm{d}, J=1.0 \mathrm{~Hz})$, 7.28 (1H, dd, $J=8.4$ \& $1.0 \mathrm{~Hz}), 6.85(2 \mathrm{H}, \mathrm{d}, J=7.6 \mathrm{~Hz}), 4.18(2 \mathrm{H}, \mathrm{t}, J=7.6 \mathrm{~Hz}), 4.08$ (2H, t, $J=$ $6.4 \mathrm{~Hz}$ ), 1.86 (4H, quint, $J=7.2 \mathrm{~Hz}), 1.25-1.54(20 \mathrm{H}, \mathrm{m}), 0.89(3 \mathrm{H}, \mathrm{t}, J=4.4 \mathrm{~Hz}), 0.87$ (3H, t, $J=$ $5.6 \mathrm{~Hz})$.

MS m/z (EI): 487, 486, $485\left(\mathrm{M}^{+}\right)$.

\section{[N-octyl-7-(octyloxy)-carbazol-2-yl]boronic acid (16)}

A solution of $2.5 \mathrm{~mol} / \mathrm{L} n$-BuLi in hexanes $\left(12.34 \mathrm{~cm}^{3}\right) \mathrm{x}$ mmol was added drop-wise to a dry THF $\left(150 \mathrm{~cm}^{3}\right)$ solution of 2-bromo- $N$-octyl-7-(octyloxy)-carbazole $(10.00 \mathrm{~g}, 20.56 \mathrm{mmol})$ at $-78^{\circ} \mathrm{C}$, and the resultant mixture was stirred for $1 \mathrm{~h}$ at $-78^{\circ} \mathrm{C}$. Then 2-triisopropyl borate $\left(9.5 \mathrm{~cm}^{3}, 41.12 \mathrm{mmol}\right.$, $0.815 \mathrm{~g} \mathrm{~cm}^{-3}$ ) was added drop-wise to the reaction mixture, which was then stirred for $1 \mathrm{~h}$ at $-78{ }^{\circ} \mathrm{C}$, allowed to warm to room temperature and then stirred overnight. Hydrochloric acid $\left(34.26 \mathrm{~cm}^{3}, 12\right.$ mol/L)x mmol was added to the reaction mixture. The resultant mixture stirred for $1 \mathrm{~h}$ and then diluted with water $\left(50 \mathrm{~cm}^{3}\right)$ and extracted with diethyl ether $\left(3 \times 100 \mathrm{~cm}^{3}\right)$. The combined organic extracts were washed with water $\left(2 \times 100 \mathrm{~cm}^{3}\right)$, dried $\left(\mathrm{MgSO}_{4}\right)$, filtered and concentrated down under reduced pressure. The crude product was purified via washing with hexane $\left(2 \times 100 \mathrm{~cm}^{3}\right)$ and then dried under reduced pressure to give the desired product as a white solid (8.58 g, $92 \%$ ).

Melting Point $/{ }^{\circ} \mathrm{C}: 127-128$.

${ }^{1} \mathrm{H}$ NMR $\left(\mathrm{CDCl}_{3}\right) \delta_{\mathrm{H}}: 8.33(1 \mathrm{H}, \mathrm{s}), 8.18(1 \mathrm{H}, \mathrm{t}, J=7.8 \mathrm{~Hz}), 7.96-8.06(2 \mathrm{H}, \mathrm{m}), 7.79(1 \mathrm{H}, \mathrm{s}), 7.50$

(1H, d, $J=7.2 \mathrm{~Hz}), 6.91$ (2H, d, $J=8.0 \mathrm{~Hz}), 4.32(2 \mathrm{H}, \mathrm{t}, J=7.2 \mathrm{~Hz}), 4.08(2 \mathrm{H}, \mathrm{t}, J=6.8 \mathrm{~Hz}), 1.80$

(4H, quint, $J=7.2 \mathrm{~Hz}), 1.24-1.50(20 \mathrm{H}, \mathrm{m}), 0.86(3 \mathrm{H}, \mathrm{t}, J=6.8 \mathrm{~Hz}), 0.83(3 \mathrm{H}, \mathrm{t}, J=6.4 \mathrm{~Hz})$. 
MS m/z (EI): 452, 451(M+1), 450.

\section{2,7- bis(Thien-2-yl)-9,9-dipropyl-fluorene (18)}

A mixture of $\mathrm{Pd}\left(\mathrm{PPh}_{3}\right)_{4}$ (0.30 g, $\left.0.26 \mathrm{mmol}\right)$, 2,7-dibromo-9,9-dipropyl-fluorene (5.00 g, 12.20 mmol) and 2-(tributylstannyl)thiophene $(11.38 \mathrm{~g}, 30.50 \mathrm{mmol})$ in DMF $\left(100 \mathrm{~cm}^{3}\right)$ was stirred and heated to $90{ }^{\circ} \mathrm{C}$ overnight. Then, the cooled reaction mixture was washed with water $\left(100 \mathrm{~cm}^{3}\right)$ and then extracted with DCM $\left(3 \times 100 \mathrm{~cm}^{3}\right)$. The combined organic layers were washed with water $(2$ $\left.\times 100 \mathrm{~cm}^{3}\right)$, dried over magnesium sulfate $\left(\mathrm{MgSO}_{4}\right)$, filtered and then concentrated under reduced pressure. The crude product was purified using column chromatography $\left[\mathrm{K}_{2} \mathrm{CO}_{3} /\right.$ silica 1:9, DCM/Hexane 5:10] to remove the tin side products and then re-crystallised from EtOH/DCM to afford the desired product as a light yellow solid (3.98 g, $79 \%$ ).

Melting Point $/{ }^{\circ} \mathrm{C}: 168-170$.

${ }^{1} \mathrm{H}$ NMR $\left(\mathrm{CDCl}_{3}\right) \delta_{\mathrm{H}}: 7.66(2 \mathrm{H}, \mathrm{d}, J=8.0 \mathrm{~Hz}), 7.59(2 \mathrm{H}, \mathrm{dd}, J=8.0 \& 1.6 \mathrm{~Hz}), 7.55(2 \mathrm{H}, \mathrm{d}, J=$ $2.0 \mathrm{~Hz}), 7.37(2 \mathrm{H}, \mathrm{d}, J=3.8 \mathrm{~Hz}), 7.28(2 \mathrm{H}, \mathrm{d}, J=5.2 \mathrm{~Hz}), 7.10(2 \mathrm{H}, \mathrm{dd}, J=7.8 \& 1.2 \mathrm{~Hz}), 2.00$ (4H, m), 0.66-0.70 (10H, m).

MS m/z (EI): 416, 415, 414 (M+ $\mathrm{M}^{+}$M100).

\section{2,7-bis-(5-Bromothiophen-2-yl)-9,9-dipropyl-fluorene (19)}

$\mathrm{N}$-bromosuccinimide (NBS) (0.95 g, $5.32 \mathrm{mmol}$ ) was added in a small portion into a solution of 2,7bis(thien-2-yl)-9,9-dipropyl-fluorene $(1.00 \mathrm{~g}, 2.42 \mathrm{mmol})$ in acetic acid $\left(10 \mathrm{~cm}^{3}\right)$ and chloroform $\left(10 \mathrm{~cm}^{3}\right)$ in the dark. The resultant mixture was stirred overnight at room temperature. The reaction mixture was poured into water $\left(100 \mathrm{~cm}^{3}\right)$ and then extracted with DCM $\left(3 \times 100 \mathrm{~cm}^{3}\right)$. The combined organic layers were washed with water $\left(2 \times 100 \mathrm{~cm}^{3}\right)$, dried over magnesium sulfate $\left(\mathrm{MgSO}_{4}\right)$, filtered and then concentrated under reduced pressure. The crude product was purified using column chromatography [Hexane] to afford the desired product as a yellow solid (0.95 g, 77 \%).

Melting Point $/{ }^{\circ} \mathrm{C}: 165-166$.

${ }^{1} \mathrm{H}$ NMR $\left(\mathrm{CDCl}_{3}\right) \delta_{\mathrm{H}}: 7.65(2 \mathrm{H}, \mathrm{d}, J=8.0 \mathrm{~Hz}), 7.48(2 \mathrm{H}, \mathrm{dd}, J=8.0 \& 1.6 \mathrm{~Hz}), 7.44(2 \mathrm{H}, \mathrm{d}, J=$ $1.6 \mathrm{~Hz}), 7.10$ (2H, d, $J=4.0 \mathrm{~Hz}), 7.04$ (2H, d, $J=3.6 \mathrm{~Hz}), 1.97$ (4H, m), 0.66-0.69 (10H, m). MS m/z (EI): 574, $572\left(\mathrm{M}^{+}\right), 570$. 


\section{5,5'-bis-[9-Octyl-7-(octyloxy)-carbazol-2-yl]-2,2'-bithiophene (1)}

A mixture of $\mathrm{Pd}\left(\mathrm{PPh}_{3}\right)_{4}$ (0.01g, $\left.0.008 \mathrm{mmol}\right)$, 5,5'-dibromo-2,2'-bithiophene (0.20 g, $\left.0.618 \mathrm{mmol}\right)$, [N-octyl-7-(octyloxy)-carbazol-2-yl]boronic acid (0.84 g, 1.85 mmol), $\mathrm{K}_{2} \mathrm{CO}_{3}(0.52 \mathrm{~g}, 3.71 \mathrm{mmol})$ and $\mathrm{H}_{2} \mathrm{O}\left(2 \mathrm{~cm}^{3}\right)$ in DMF $\left(30 \mathrm{~cm}^{3}\right)$ was heated at $80{ }^{\circ} \mathrm{C}$ overnight. The cooled reaction mixture was washed with water $\left(100 \mathrm{~cm}^{3}\right)$ and extracted with DCM $\left(3 \times 100 \mathrm{~cm}^{3}\right)$. The combined organic layers were washed with water $\left(2 \times 100 \mathrm{~cm}^{3}\right)$, dried $\left(\mathrm{MgSO}_{4}\right)$, filtered and then concentrated down under reduced pressure. The residue was purified using column chromatography [silica, DCM/hexane 5:10] to afford the desired product as an yellow solid $(0.49 \mathrm{~g}, 82 \%)$.

Melting Point $/{ }^{\circ} \mathrm{C}:$ Cr 136 N 151 I.

${ }^{1} \mathrm{H}$ NMR $\left(\mathrm{CDCl}_{3}\right) \delta_{\mathrm{H}}: 7.96(2 \mathrm{H}, \mathrm{d}, J=8.0 \mathrm{~Hz}), 7.93(2 \mathrm{H}, \mathrm{d}, J=8.4 \mathrm{~Hz}), 7.53(2 \mathrm{H}, \mathrm{s}), 7.48$ (2H, d, $J=8.0 \mathrm{~Hz}), 7.32(2 \mathrm{H}, \mathrm{d}, J=4.0 \mathrm{~Hz}), 7.23(2 \mathrm{H}, \mathrm{d}, J=4.0 \mathrm{~Hz}), 6.85(4 \mathrm{H}, \mathrm{dd}, J=7.2 \& 2.0 \mathrm{~Hz})$, $4.27(4 \mathrm{H}, \mathrm{t}, J=6.8 \mathrm{~Hz}), 4.10(4 \mathrm{H}, \mathrm{t}, J=6.4 \mathrm{~Hz}), 1.90(8 \mathrm{H}, \mathrm{m}), 1.26-1.53(40 \mathrm{H}, \mathrm{m}), 0.91(6 \mathrm{H}, \mathrm{t}, J$ $=7.6 \mathrm{~Hz}), 0.87(6 \mathrm{H}, \mathrm{t}, J=7.2 \mathrm{~Hz})$.

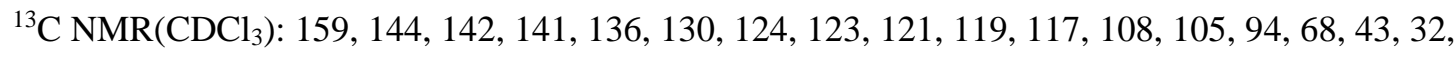
30, 29, 26, 23, 14.

IR $v_{\max } / \mathrm{cm}^{-1}:$ 2922, 2850, 1599, 1499, 1455, 1439, 1390, 1347, 1248, 1218, 1208, 1167, 1145, 1126, 1056, 935, 880, 839, 801, 786, 768.

MS m/z (MALDI): 979, 978, 977 (M+, M100).

Combustion analysis:

Expected: C, 78.64\%; H, 8.66\%; N, 2.87\%; S, 6.56\%.

Obtained: C, 78.62\%; H, 8.70\%; N, 2.83\%; S, 6.66\%

\section{7,7'-(9,9-Dioctyl-fluorene-2,7-diyl)-bis-[9-octyl-7-(octyloxy)-carbazole] (2)}

A mixture of $\mathrm{Pd}\left(\mathrm{PPh}_{3}\right)_{4}$ (0.01g, $\left.0.008 \mathrm{mmol}\right)$, 2,7-dibromo-9,9-dioctyl-fluorene (0.20 g, 0.365 mmol), [N-octyl-7-(octyloxy)-carbazol-2-yl]boronic acid (0.50 g, 1.10 mmol), $\mathrm{K}_{2} \mathrm{CO}_{3}(0.31 \mathrm{~g}, 2.20$ mmol) and $\mathrm{H}_{2} \mathrm{O}\left(2 \mathrm{~cm}^{3}\right)$ in DMF $\left(30 \mathrm{~cm}^{3}\right)$ was heated at $80{ }^{\circ} \mathrm{C}$ overnight. The cooled reaction mixture was washed with water $\left(100 \mathrm{~cm}^{3}\right)$ and then extracted with DCM $\left(3 \times 100 \mathrm{~cm}^{3}\right)$. The combined organic layers were washed with water $\left(2 \times 100 \mathrm{~cm}^{3}\right)$, dried over magnesium sulfate $\left(\mathrm{MgSO}_{4}\right)$, filtered and then concentrated down under reduced pressure. The residue was purified using column chromatography [silica, DCM/Hexane 3:10] to afford the desired product as a pale- 
yellow solid (0.32 g, $73 \%)$.

Melting Point $/{ }^{\circ} \mathrm{C}: 66$.

${ }^{1} \mathrm{H} \mathrm{NMR}\left(\mathrm{CDCl}_{3}\right) \delta_{\mathrm{H}}: 8.06(2 \mathrm{H}, \mathrm{d}, J=8.0 \mathrm{~Hz}), 7.98(2 \mathrm{H}, \mathrm{d}, J=8.4 \mathrm{~Hz}), 7.83(2 \mathrm{H}, \mathrm{d}, J=8.0 \mathrm{~Hz})$, $7.71(2 \mathrm{H}, \mathrm{d}, J=8.0 \mathrm{~Hz}), 7.68(2 \mathrm{H}, \mathrm{s}), 7.59(2 \mathrm{H}, \mathrm{s}), 7.53(2 \mathrm{H}, \mathrm{d}, J=8.0 \mathrm{~Hz}), 6.88$ (4H, dd, $J=8.0$ \& $2.0 \mathrm{~Hz}), 4.32(4 \mathrm{H}, \mathrm{t}, J=6.8 \mathrm{~Hz}), 4.12(4 \mathrm{H}, \mathrm{t}, J=6.4 \mathrm{~Hz}), 3.92(4 \mathrm{H}, \mathrm{m}), 1.94(8 \mathrm{H}, \mathrm{m}), 1.10-1.48$ (60H, m), 0.90 (12H, t, $J=7.2 \mathrm{~Hz}), 0.75-0.84(10 \mathrm{H}, \mathrm{m})$.

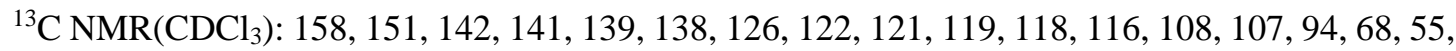
43, 40, 32, 29, 23, 14.

IR $v_{\max } / \mathrm{cm}^{-1}: 2920,2850,1601,1499,1460,1389,1352,1245,1169,1126,1060,1022,989,872$, 768.

MS m/z (MALDI): 1204, 1203, $1202\left(\mathrm{M}^{+}, \mathrm{M} 100\right)$.

Combustion analysis:

Expected: C, 84.94\%; H, 10.06\%; N, 2.33\%

Obtained: C, 84.96\%; H, 10.02\%; N, 2.36\%

\section{3,7-bis-[9-Octyl-7-(octyloxy)-carbazol-2-yl]dibenzo[b,d]thiophene (3)}

A mixture of $\mathrm{Pd}\left(\mathrm{PPh}_{3}\right)_{4}(0.01 \mathrm{~g}, 0.008 \mathrm{mmol})$, 3,7-dibromodibenzo[b,d]thiophene $(0.20 \mathrm{~g}, 0.60$ mmol), [ $N$-octyl-7-(octyloxy)-carbazol-2-yl]boronic acid (0.80 g, 1.76 mmol), $\mathrm{K}_{2} \mathrm{CO}_{3}(0.50 \mathrm{~g}, 3.60$ mmol) and $\mathrm{H}_{2} \mathrm{O}\left(2 \mathrm{~cm}^{3}\right)$ in DMF $\left(30 \mathrm{~cm}^{3}\right)$ was heated at $80{ }^{\circ} \mathrm{C}$ overnight. The cooled reaction mixture was washed with water $\left(100 \mathrm{~cm}^{3}\right)$, and extracted with DCM $\left(3 \times 100 \mathrm{~cm}^{3}\right)$. The combined organic layers were washed with water $\left(2 \times 100 \mathrm{~cm}^{3}\right)$, dried over magnesium sulfate, filtered and concentrated under reduced pressure. The residue was purified using column chromatography [silica, DCM/Hexane 4:10] to afford the desired product as a pale-yellow solid (0.49 g, 86 \%).

Melting Point $/{ }^{\circ} \mathrm{C}:$ Cr 141 N 202 I.

${ }^{1} \mathrm{H}$ NMR $\left(\mathrm{CDCl}_{3}\right) \delta_{\mathrm{H}}: 8.26(2 \mathrm{H}, \mathrm{d}, J=8.4 \mathrm{~Hz}), 8.19(2 \mathrm{H}, \mathrm{d}, J=1.0 \mathrm{~Hz}), 8.07(2 \mathrm{H}, \mathrm{d}, J=8.0 \mathrm{~Hz})$, $7.98(2 \mathrm{H}, \mathrm{d}, J=8.4 \mathrm{~Hz}), 7.84(2 \mathrm{H}, \mathrm{dd}, J=8.0 \& 1.0 \mathrm{~Hz}), 7.62(2 \mathrm{H}, \mathrm{s}), 7.55(2 \mathrm{H}, \mathrm{dd}, J=6.8 \& 1.0$ Hz), 6.88 (4H, d, $J=8.4 \mathrm{~Hz}$ ), $4.32(4 \mathrm{H}, \mathrm{t}, J=7.2 \mathrm{~Hz}), 4.11$ (4H, t, $J=6.4 \mathrm{~Hz}), 1.93(8 \mathrm{H}, \mathrm{m}), 1.24-$ $1.52(40 \mathrm{H}, \mathrm{m}), 0.90(12 \mathrm{H}, \mathrm{t}, J=6.8 \mathrm{~Hz})$.

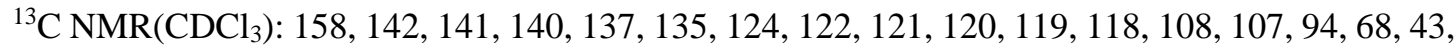
$32,29,23,14$ 
IR $v_{\max } / \mathrm{cm}^{-1}: 2919,2850,1601,1499,1453,1436,1392,1354,1247,1190,1177,1127,1045,992$, 872, 797, 768.

MS m/z (MALDI): 996, 995 (M+1, M100).

Combustion analysis:

Expected: C, 82.04\%; H, 8.71\%; N, 2.81\%; S, 3.22\%

Obtained: C, 82.12\%; H, 8.73\%; N, 2.72\%; S, 3.24\%

\section{4,7-bis-[9-Octyl-7-(octyloxy)-carbazol-2-yl]benzo[c][1,2,5]thiadiazole (4)}

A mixture of $\mathrm{Pd}\left(\mathrm{PPh}_{3}\right)_{4}$ (0.01g, $\left.0.008 \mathrm{mmol}\right)$, 4,7-dibromo-2,1,3-benzothiadiazole (0.20 g, 0.681 mmol), [ $N$-octyl-7-(octyloxy)-carbazol-2-yl]boronic acid (0.93 g, 2.04 mmol), $\mathrm{K}_{2} \mathrm{CO}_{3}(0.57 \mathrm{~g}, 4.08$ mmol) and $\mathrm{H}_{2} \mathrm{O}\left(2 \mathrm{~cm}^{3}\right)$ in DMF $\left(30 \mathrm{~cm}^{3}\right)$ was heated at $80{ }^{\circ} \mathrm{C}$ overnight. The cooled reaction mixture was washed with water $\left(100 \mathrm{~cm}^{3}\right)$, and extracted with DCM $\left(3 \times 100 \mathrm{~cm}^{3}\right)$. The combined organic layers were washed with water $\left(2 \times 100 \mathrm{~cm}^{3}\right)$, dried over magnesium sulfate $\left(\mathrm{MgSO}_{4}\right)$, filtered and then concentrated down under reduced pressure. The residue was purified using column chromatography [silica, DCM/Hexane 5:10] to afford the desired product as a red solid (0.60 g, $93 \%)$.

Melting Point $/{ }^{\circ} \mathrm{C}$ : Tg 85; Cr 105 I (Heating); I 94 N (Cooling).

${ }^{1} \mathrm{H} \mathrm{NMR}\left(\mathrm{CDCl}_{3}\right) \delta_{\mathrm{H}}: 8.14(2 \mathrm{H}, \mathrm{d}, J=8.0 \mathrm{~Hz}), 8.04(2 \mathrm{H}, \mathrm{s}), 8.01(2 \mathrm{H}, \mathrm{d}, J=8.4 \mathrm{~Hz}), 7.93(2 \mathrm{H}, \mathrm{s})$, 7.80 (2H, dd, $J=8.0 \& 1.0 \mathrm{~Hz}), 6.89(4 \mathrm{H}, \mathrm{d}, J=8.4 \mathrm{~Hz}), 4.34(4 \mathrm{H}, \mathrm{t}, J=7.2 \mathrm{~Hz}), 4.12(4 \mathrm{H}, \mathrm{t}, J=$ $6.8 \mathrm{~Hz}), 1.96(8 \mathrm{H}, \mathrm{m}), 1.22-1.52(40 \mathrm{H}, \mathrm{m}), 0.90(6 \mathrm{H}, \mathrm{t}, J=6.8 \mathrm{~Hz}), 0.84(6 \mathrm{H}, \mathrm{t}, J=7.2 \mathrm{~Hz})$.

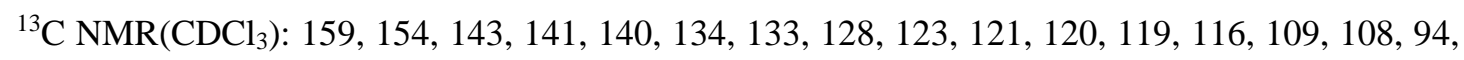
68, 43, 32, 29, 26, 14.

IR $v_{\max } / \mathrm{cm}^{-1}: 2917,2849,1600,1499,1455,1439,1362,1257,1211,1177,1130,1056,982,895$, 819, 801, 766.

MS m/z (MALDI): 948, 947 (M+, M100).

Combustion analysis:

Expected: C, 78.60\%; H, 8.72\%; N, 5.91\%;S, 3.38\%

Obtained: C, 78.62\%; H, 8.73\%; N, 5.91\%; S, 3.30\% 
A mixture of $\mathrm{Pd}\left(\mathrm{PPh}_{3}\right)_{4}$ (0.01g, 0.008 mmol), 2,7-bis-(5-bromothiophen-2-yl)-9,9-dipropylfluorene (0.20 g, 0.35 mmol), [ $N$-octyl-7-(octyloxy)-carbazol-2-yl]boronic acid (0.48 g, 1.05 mmol), $\mathrm{K}_{2} \mathrm{CO}_{3}(0.30 \mathrm{~g}, 2.10 \mathrm{mmol})$ and $\mathrm{H}_{2} \mathrm{O}\left(2 \mathrm{~cm}^{3}\right)$ in DMF $\left(30 \mathrm{~cm}^{3}\right)$ was heated at $80{ }^{\circ} \mathrm{C}$ overnight. The cooled reaction mixture was washed with water $\left(100 \mathrm{~cm}^{3}\right)$, and extracted with DCM $\left(3 \times 100 \mathrm{~cm}^{3}\right)$. The combined organic layers were washed with water $\left(2 \times 100 \mathrm{~cm}^{3}\right)$, dried over magnesium sulfate $\left(\mathrm{MgSO}_{4}\right)$, filtered and then concentrated down under reduced pressure. The residue was purified using column chromatography [silica, DCM/Hexane 4:10] to afford the desired product as a yellow solid (0.36 g, $75 \%)$.

Melting Point/ ${ }^{\circ} \mathrm{C}: \mathrm{Tg} 32 \mathrm{Cr}-\mathrm{N} 161 \mathrm{I}$.

${ }^{1} \mathrm{H}$ NMR $\left(\mathrm{CDCl}_{3}\right) \delta_{\mathrm{H}}: 7.99(2 \mathrm{H}, \mathrm{d}, J=8.0 \mathrm{~Hz}), 7.94(2 \mathrm{H}, \mathrm{d}, J=8.4 \mathrm{~Hz}), 7.72(2 \mathrm{H}, \mathrm{d}, J=7.6 \mathrm{~Hz})$, $7.68(2 \mathrm{H}, \mathrm{d}, J=7.6 \mathrm{~Hz}), 7.63(2 \mathrm{H}, \mathrm{d}, J=1.0 \mathrm{~Hz}), 7.59(2 \mathrm{H}, \mathrm{d}, J=1.0 \mathrm{~Hz}), 7.54(2 \mathrm{H}, \mathrm{dd}, J=8.0$ \& $1.0 \mathrm{~Hz}), 7.41(4 \mathrm{H}, \mathrm{dd}, J=6.0 \& 2.4 \mathrm{~Hz}), 6.86(4 \mathrm{H}, \mathrm{dd}, J=7.6 \& 2.0 \mathrm{~Hz}), 4.28(4 \mathrm{H}, \mathrm{t}, J=7.2$ Hz), $4.11(4 \mathrm{H}, \mathrm{t}, J=6.8 \mathrm{~Hz}), 2.07(4 \mathrm{H}, \mathrm{m}), 1.91(8 \mathrm{H}, \mathrm{m}), 1.26-1.52(40 \mathrm{H}, \mathrm{m}), 0.90$ (12H, t, $J=7.2$ $\mathrm{Hz}), 0.72-0.83(10 \mathrm{H}, \mathrm{m})$.

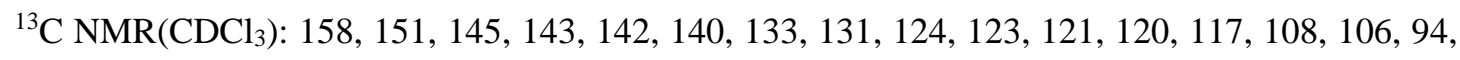
68, 56, 43, 32, 29, 27, 26, 23, 14.

IR $v_{\max } / \mathrm{cm}^{-1}: 2921,2851,1601,1501,1458,1390,1347,1247,1189,1170,1125,1053,1021,993$, 872, 769.

MS m/z (MALDI): 1228, 1227, $1226\left(\mathrm{M}^{+}\right)$.

Combustion analysis:

Expected: C, 81.32\%; H, 8.55\%; N, 2.29\%; S, 5.23\%.

Obtained: C, 81.32\%; H, 8.58\%; N, 2.40\%; S, 5.14\%

\section{Results and Discussion}

\subsection{Synthetic Discussion of Reaction Scheme 1}

The synthesis and application of carbazole-functional derivatives have been of great interest in materials chemistry, especially in organic electronics, due to their intrinsic photo-physical and redox properties, e.g. relatively intense luminescence and reversible oxidation processes, $\pi$-stacking capability and ease of further derivation [44-51]. Therefore, a series of carbazole-functionalised 
derivatives containing different cores were synthesised and are discussed below.

1-(Octyloxy)-4-iodobenzene (11) was synthesised in excellent yield (95\%) by reacting 4iodophenol (10) with 1-bromooctane, using $\mathrm{K}_{2} \mathrm{CO}_{3}$, with $\mathrm{DMF}$ as the solvent at $120^{\circ} \mathrm{C}$. An Ullmann cross-coupling reaction was carried out between 2,5-dibromonitrobenzene (12) and compound 11 using copper powder as catalyst at $175^{\circ} \mathrm{C}$ to give 4-bromo-4'-octyloxy-2-nitrobiphenyl (13) in good yield (69\%). The 2-bromo-7-octyloxycarbazole (14) was obtained from the ring closure reaction of compound 13 with triethyl phosphite in good yield (78\%). Given that solid triphenyl phosphine is safer and less harmful than liquid triethyl phosphite, we tried triphenyl phosphine instead of triethyl phosphite, giving an approximate equal yield of $76 \%$. However, purification of the product was less efficient using triphenyl phosphine as column chromatography had to be carried out with more solvent consumption, due to the excess quantity of triphenyl phosphine (up to $100 \%$ excess), with similar yields obtained. In such cases, we tried to use $25 \%$ and $50 \%$ excess of triphenyl phosphine for the ring closing reaction of compound $\mathbf{1 3}$ to decrease by-products and facilitate purification, but this resulted in a very poor yield. Therefore, liquid triethyl phosphite was chosen and the result showed easier purification and good yield. Since triethyl phosphite is more harmful than triphenyl phosphine, all reaction procedures and purification were performed in a fume cupboard. Compound 14 was alkylated using commercially available 1-bromooctane, using $\mathrm{NaH}$, with DMF as the solvent to give 2-bromo-9-octyl-7-octyloxy-carbazole (15) in excellent yield (99\%). Commercially available 2-triisopropyl borate was used to treat the lithiated compound $\mathbf{3 6}$ with $n$-butylithium at $78{ }^{\circ} \mathrm{C}$. Hydrolysis of the reaction mixture with aqueous hydrochloric acid gave [ $N$-octyl-7(octyloxy)-carbazol-2-yl]boronic acid (16) in excellent yield (92\%). A Stille aryl-aryl, crosscoupling reaction was carried out using 2,7-dibromo-9,9-dipropyl-fluorene (17), 2(tributylstannyl)thiophene, tetrakis(triphenylphosphine)palladium(0) as catalyst, and DMF as solvent to afford symmetrical bis-thiophene intermediate 18 in good yield (79\%). The position 5 of two thiophene rings of compound $\mathbf{1 8}$ was then brominated using NBS as brominating agent in acetic acid and chloroform as solvent in the dark to give 2,7-bis-(5-bromothiophen-2-yl)-9,9-dipropylfluorene (19) in good yield (77\%). Dibrominated aromatic cores 5,5'-dibromo-2.2'-bithiophene (20), 2,7-dibromo-9,9-dioctyl-fluorene (21), 3,7-dibromodibenzo[b,d]thiophene (22), and 4,7-Dibromo2,1,3-benzothiadiazole (23), 2,7-bis-(5-bromothiophen-2-yl)-9,9-dipropyl-fluorene (19) were 
reacted with the same boronic acid $\mathbf{1 6}$ in a Suzuki aryl-aryl cross-coupling reaction using $\mathrm{Pd}\left(\mathrm{PPh}_{3}\right)_{4}$ as catalyst, $\mathrm{K}_{2} \mathrm{CO}_{3}$ aqueous as base and DMF as solvent to give 5,5'-bis-[9-octyl-7-(octyloxy)carbazol-2-yl]-2,2'-bithiophene (1) (82\%), 7,7'-(9,9-dioctyl-fluorene-2,7-diyl)-bis-[9-octyl-7-

(octyloxy)-carbazole]

yl]dibenzo[b,d]thiophene

(73\%),

(86\%),
3,7-bis-[9-Octyl-7-(octyloxy)-carbazol-2-

4,7-bis-[9-Octyl-7-(octyloxy)-carbazol-2-

yl]benzo[c][1,2,5]thiadiazole (4) (93\%), 2,7-[bis(thien-2-yl)-9,9-dipropyl-fluorene-5,5-diyl]-bis[9-octyl-7-(octyloxy)-carbazole] (5) (75\%), respectively.

\subsection{Liquid Crystalline Oligomer Properties and Their Opto-electronic Application}

The chemical structures, transition temperatures, energy levels and band gaps of the oligomers 1-5 are collated in Table 1 to facilitate a comparison of the mesomorphic and physical characteristics of these compounds differing only in the nature of the central core of their molecular structure. The data listed in Table 1 should also serve as background information in evaluating a potential correlation between the mesomorphic behavior and liquid crystalline transition temperature of these compounds and their relative performance as emissive materials in test OLEDs. The data collated in Table 1 reveals a direct correlation between the length-to-breadth ratio of the molecular core and the value for the nematic clearing point. For example a high clearing point $\left(202^{\circ} \mathrm{C}\right)$ and a very broad nematic phase are observed for compound 3 incorporating the rigid, planar 3,7-disubstituted dibenzo[b,d]thiophene moiety in the molecular core, whereas the analogous 9,9-dioctyl-2,7disubstituted-fluorene (compound 2) does not exhibit any observable mesomorphic behaviour. This behavior may well be due to the greater intermolecular distance between adjacent molecules generated by the two long aliphatic chains and a much lower effective length-to-breadth ratio for the molecular core. Compound $\mathbf{1}$ exhibits a narrow nematic phase $\left(15^{\circ} \mathrm{C}\right)$, with a lower clearing point (N-I) than that of either compound 3 or compound $\mathbf{5}$. The potential for inter-annular twisting between the bithiophene core and the carbazole moieties may increase the intermolecular distance between adjacent molecules. Evidence of this effect may be seen in the lower melting point of compound $\mathbf{1}$ compared to that of compound 3. Compound $\mathbf{1}$ also has a lower length-to-breadth ratio than that of either compound $\mathbf{3}$ or $\mathbf{5}$, which is probably a major factor in generating a relatively low clearing point for this material. Compound $\mathbf{4}$ is the only final product that possesses a monotropic nematic phase observed on cooling below the melting point (C-I). of $105^{\circ} \mathrm{C}$, which is probably 
caused by the steric hindrance of the 2,1,3-benzodithiadiazole core, which will, in turn, prevent close intermolecular packing between adjacent molecules leading to weaker intermolecular forces of attraction.

Figure 2 shows the Schlieren texture, with point singularities with four and two point brushes, characteristic of the nematic phase, for compound 5, observed on cooling the OPM sample from the isotropic state into glassy state. DSC analysis was used to confirm this mesomorphic behaviour. A similar Schlieren texture was observed for compounds 1, 3 and 4. A combination of OPM and DSC, see Figure 3, reveals that compound 5 possesses no observable melting point $(\mathrm{Cr}-\mathrm{N})$. Instead it softens gradually on increasing the temperature above glass transition temperature until it forms an isotropic state above the clearing point. These kinds of gradual phase change are often found with polymers, and are generally observed as a broad peak in DSC measurements [56-57]. Broad peaks were observed in the DSC measurements of $\mathbf{5}$, which is a high-molecular-weight oligomer.

OLED devices with the same layer structure, see Figure 1, but five different emissive layers were measured and compared in terms of current density, luminance and efficacy. Figure 4a shows the efficacy of the 5 devices between zero and $8 \mathrm{~V}$. Each of the OLEDs exhibits a low switch-on voltage between $2.4 \mathrm{~V}$ and 3.0 V. As Figure $4 \mathbf{b}$ shows, the luminance of each of the devices at 8.0 V differs considerably, i.e., from ca $750 \mathrm{~cd} \mathrm{~m}^{-2}$ to ca $6,000 \mathrm{~cd} \mathrm{~m}^{-2}$.

It was found that the test OLED incorporating compound $\mathbf{5}$ as the emissive layer exhibits a significantly higher efficacy and luminance than the other test devices using compounds 1, 2, 3 and 4. For example at $4 \mathrm{~V}$, it shows a luminance of $200 \mathrm{~cd} \mathrm{~m}^{-2}$ and an efficacy of $4.1 \mathrm{~cd} \mathrm{~A}^{-1}$. These reasonably high values may be attributed to the liquid crystalline nature of compound $\mathbf{5}$ and its advantageous liquid crystalline transition temperatures, i.e., compound $\mathbf{5}$ possesses a glass transition above room temperature $\left(\mathrm{Tg}=32{ }^{\circ} \mathrm{C}\right)$, and a nematic phase with a high clearing point $(\mathrm{N}-\mathrm{I}=161$ ${ }^{\circ} \mathrm{C}$ ), see Table 1. The orientational order of a nematic mesophase without a layer structure is assumed to facilitate high charge-carrier mobility by hopping [2]. The combination of a glassy nematic phase above room temperature $\left(\mathrm{Tg}=32{ }^{\circ} \mathrm{C}\right)$ and a very high clearing point $\left(\mathrm{N}-\mathrm{I}=161^{\circ} \mathrm{C}\right)$, see Table 1, for compound 5 may well result in a highly viscous nematic phase, which maintains a 
uniform structure during the OLED operation.

There is a good match between the values of the ionization potential $(-5.53 \mathrm{eV})$, see Table 1 , of compound 5 and the HOMO energy level of the hole-transport layer OTPD (IP = -5.48 eV [54]). Similarly, the electron affinity $(\mathrm{EA}=-2.89 \mathrm{eV})$ of compound 5 is similar to the LUMO energy level of electron-transporting layer of SPPO13 $(\mathrm{EA}=-2.91 \mathrm{eV})$ [55]. Figure 5 shows the energy band diagram of the materials in the OLED taking compound $\mathbf{5}$ as the emissive material. This advantageous combination of energy levels results in low charge-injection barriers for electrons and holes, respectively, relatively balanced charge injection and a corresponding high density of excitons in the emissive layer where efficient recombination can occur with the emission of light. Note that the OTPD and SPPO13 act as electron and hole blocking layers respectively, confining carriers in the emission layer and so limiting current. Further efficiency gains are likely by optimizing the thickness of the various layers in the device.

The OLED incorporating compound 2 performs poorly in terms of both efficacy and brightness and exhibits a severe efficiency roll-off with voltage. One possible reason for this poor performance may be the absence of liquid crystalline mesophases for compound 2. This OLED exhibits a higher current density than the OLED with compound $\mathbf{5}$ despite their similar IPs and compound $\mathbf{2}$ having a higher LUMO energy level $(\mathrm{EA}=-2.50 \mathrm{eV})$, which is unfavourable for electron transport. The higher current may result from leakage at crystal grain boundaries.

The OLEDs incorporating the nematic liquid crystalline compounds 1, 3 and 4 exhibit similar performance in terms of overall device efficacy. However, the devices incorporating compounds $\mathbf{1}$ and $\mathbf{4}$ have higher values of luminance and current density than those of the test OLED incorporating compound 3. The dibenzothiophene central core of compound $\mathbf{3}$ has fewer hetero-atoms than the bithiophene core of compound $\mathbf{1}$ and the benzothiadiazole core of compound $\mathbf{4}$. This leads to a bigger energy gap (3.10 eV compared to $2.60 \mathrm{eV}$ and $2.42 \mathrm{eV}$, respectively) and a higher-lying LUMO energy level (-2.47 eV compared to $-2.77 \mathrm{eV}$ and $-2.88 \mathrm{eV}$, respectively), see Table 1 . The higher energy gap and higher-lying LUMO energy level are unfavourable for efficient electron injection, thus causing an adverse effect on luminance and current density. 
The electroluminescence (EL) spectra of the five OLEDs with each compound $\mathbf{1}$ to $\mathbf{5}$ as an emissive material are shown in Figure 6. It is evident that the EL spectra from these test devices cover most of the visible spectrum, i.e., differences in the core of the molecular structure of these five test compounds gives rise to significant differences in the band gap (Eg) and the corresponding emission spectrum. The OLEDs incorporating compounds 2 and $\mathbf{3}$ exhibit blue light, while those incorporating compounds $\mathbf{1}$ and $\mathbf{5}$ emit green light and blue-green light respectively. The test device incorporating compound $\mathbf{4}$ exhibits orange light, see Figure $\mathbf{7}$ for images of working devices. However, the emission bands are broad and overlap to a significant extent, e.g., the emission of compound 5 covers the blue and green wavelengths from $450 \mathrm{~nm}$ to $550 \mathrm{~nm}$.

In addition to electrochemical analysis, time-dependent density functional theory (TD-DFT) calculations were performed to evaluate the frontier molecular orbitals and optimized geometrically molecular configuration of the compounds at the B3PW91/ 6-31 G (d) level. DFT calculations were computationally processed in theoretical gaseous conditions so that the interaction of these molecules and the solvent can be eliminated [58-59]. Figure 8 gives the electron density distributions of HOMO and LUMO for compound $\mathbf{5}$ as an example. The electron density of HOMO and LUMO is mainly localised on the sequentially aromatic fluorene and carbazole units, which show aromatic electronic $\pi-\pi^{*}$ transitions in the compound as predicted.

\section{Conclusions}

Novel nematic liquid crystalline oligomers incorporating N-heterocyclic carbazole moieties were synthesised in this work for use as electroluminescent materials in multi-stack OLEDs. Particularly, the nematic oligomer $\mathbf{5}$ proves excellent device performance due to the combined advantages of nematic mesophase with a desired glass transition temperature above room temperature $\left(32{ }^{\circ} \mathrm{C}\right)$ and high clearing point $\left(161^{\circ} \mathrm{C}\right)$, high degree of conjugation, the presence of various aromatic heterocyclic moieties, and low charge-injection barriers from the appropriate HOMO and LUMO energy levels in test OLED devices. The design of different aromatic cores also results in compounds with different electroluminescent colours. 


\section{Acknowledgments}

The China Scholarship Council and the University of Hull are thanked for their support of a $\mathrm{PhD}$ studentship for Guang Hu.

\section{References}

[1] Zhang S, Yao L, Peng Q, et al. Achieving a significantly increased efficiency in nondoped pure blue fluorescent OLED: a quasi-equivalent hybridized excited state. Adv Funct Mater. 2015;25(11):1755-1762.

[2] Kelly SM. Flat Panel Display: Advanced Organic Materials. Cambridge (UK). The Royal Society of Chemistry; 2000.

[3] Chiang CJ, Kimyonok A, Etherington MK, et al. Ultrahigh efficiency fluorescent single and bi-layer organic light emitting diodes: the key role of triplet fusion. Adv Funct Mater. 2013;23(6):739-746.

[4] Uoyama H, Goushi K, Shizu K, et al. High efficient organic light-emitting diodes from delayed fluorescence. Nature. 2012;492: 234-238.

[5] Xu W, Zhao J, Qian L, et al. Sorting of large-diameter semiconducting carbon nanotube and printed flexible driving circuit for organic light emitting diodes (OLED). Nanoscale. 2014;6:1589-1595.

[6] Choi YJ, Gong SC, Kang KM, et al. Enhanced hole injection into indium-free organic lightemitting diodes by fluorine-doping-induced texturing of a zinc oxide surface. J Mater Chem C. 2014;2:8344-8349.

[7] Chen Y, Ma D. Organic semiconductor heterojunctions as charge generation layers and their application in tandem organic light-emitting diodes for high power efficiency. J Mater Chem. 2012;22:18718-18734.

[8] Hofmann S, Thomschke M, Lussem B, et al. Top-emitting organic light-emitting diodes. Opt Express. 2011;19(6):A1250-A1264.

[9] Cha SJ, Cho SN, Lee WH, et al. Thermally cross-linkable hole transport polymers for solution-based organic light-emitting diodes. Macromol Rapid Commun. 2014; 35:807-812.

[10] Yook KS, Lee JY. Organic materials for deep blue phosphorescent organic light-emitting diodes. Adv Mater. 2012;24:3169-3190. 
[11] Hudson ZM, Wang Z, Helander MG, et al. N-heterocyclic carbazole-based hosts for simplified single-layer phosphorescent OLEDs with high efficiencies. Adv Mater. 2012;24:2922-2928.

[12] Yuan WZ, Gong Y, Chen S, et al. Efficient solid emitters with aggregation-induced emission and intramolecular charge transfer characteristics: molecular design, synthesis, photophysical behaviors, and OLED application. Chem Mater. 2012;24(8):1518-1528.

[13] Liu X, Sun Y, Hsu BBY, et al. Design and properties of intermediate-sized narrow bandgap conjugated molecules relevant to solution-processed organic solar cells. J Am Chem Soc. 2014;136(15):5697-5708.

[14] Wu W, Liu Y, Zhu D. $\pi$-Conjugated molecules with fused rings for organic field-effect transistors: design, synthesis and applications. Chem Soc Rev. 2010;39:1489-1502.

[15] Yan H, Chen Z, Zheng Y, et al. A high-mobility electron-transporting polymer for printed transistors. Nature. 2009;457:679-686.

[16] Brunner K, Dijken A, Borner H, et al. Carbazole compounds as host materials for triplet emitters in organic light-emitting diodes: tuning the HOMO level without influencing the triplet energy in small molecules. J Am Chem Soc. 2004;126(19):6035-6042.

[17] Duan L, Hou L, Lee TW, et al. Solution processable small molecules for organic lightemitting diodes. J Mater Chem. 2010;20:6392-6407.

[18] Duan L, Qiao J, Sun Y, et al. Strategies to design bipolar small molecules for OLEDs: donor-acceptor structure and non-donor-acceptor structure. Adv Mater. 2011;23(9):11371144.

[19] Okamoto K, Zhang J, Housekeeper JB, et al. C-H arylation reaction: atom efficient and greener syntheses of $\pi$-conjugated small molecules and macromolecules for organic electronic materials. Macromolecules. 2013;46(20):8059-8078.

[20] Thiery S, Tondelier D, Declairieux C, et al. 9, 9'-Spirobifluorene and 4-phenyl-9, 9'spirobifluorene: pure hydrocarbon small molecules as hosts for efficient green and blue PhOLEDs. J Mater Chem C. 2014;2:4156-4166.

[21] Burroughes JH, Bradley DDC, Brown AR, et al. Light-emitting diodes based on conjugated polymers. Nature. 1990;347:539-541.

[22] He Y, Gong S, Hattori R, et al. High performance organic polymer light-emitting 
heterostructure devices. Appl Phys Lett. 1999;74(16):2265-2267.

[23] Aldred MP, Eastwood AJ, Kelly SM, et al. Light-emitting fluorine photoreactive liquid crystals for organic electroluminescence. Chem Mater. 2004;16(24):4928-4936.

[24] Tsoi WC, O’Neill M, Aldred MP, et al. Distributed bilayer photovoltaics based on nematic liquid crystal polymer networks. Chem Mater. 2007;19(23):5475-5484.

[25] Wang H, Xu Y, Tsuboi T, et al. Energy transfer in polyfluorene copolymer used for whitelight organic emitting devices. Organic Electronics. 2013;14(3):827-838.

[26] Osken I, Gundogan AS, Tekin E, et al. Fluorene-dithienothiophene-S, S-dioxide copolymers. Fine-tuning for OLED applications. Macromolecules. 2013;46(23):9202-9210.

[27] Hofle S, Schienle A, Bruns M, et al. Enhanced electron injection into inverted polymer light-emitting diodes by combined solution-processed zinc oxide/polyethylenimine interlayers. Adv Mater. 2014;26(17):2750-2754.

[28] Li G, Tan ZK, Di D, et al. Efficient light-emitting diodes based on nanocrystalline perovskite in a dielectric polymer matrix. Nano Lett. 2015;15(4):2640-2644.

[29] Nikolaenko AE, Cass M, Bourcet F, et al. Thermally activated delayed fluorescence in polymers: a new route toward highly efficient solution processable OLEDs. Adv Mater. 2015;27(44):7236-7240.

[30] Pandya SU, Attar HAA, Jankus V, et al. Solution-processable ambipolar host oligomers with high triplet energies for phosphorescent green emitters. J Mater Chem. 2011;21:18439-18446.

[31] Ananthakrishnan SJ, Varathan E, Ravindran E, et al. A solution processable fluorinefluorenone oligomer with aggregation induced emission enhancement. Chem Commun. 2013;49:10742-10744.

[32] Sasabe H, Toyota N, Nakanishi H, et al. 3, 3'-Bicarbazole-based host materials for highefficiency blue phosphorescent OLEDs with extremely low driving voltage. Adv Mater. 2012;24(24):3212-3217.

[33] Chen K, Zhao HR, Fan ZK, et al. Macrospirocyclic oligomer based on triphenylamine and diphenylphosphine oxide as a bipolar host for efficient blue electrophosphorescent organic light-emitting diodes (OLEDs). Org Lett. 2015;17(6):1413-1416.

[34] Senes A, Meskers SCJ, Dijkstra WM, et al. Transition dipole moment orientation in films 
of solution processed fluorescent oligomers: investigating the influence of molecular anisotropy. J Mater Chem C. 2016;4:6302-6308.

[35] Burn PL, Bradley DDC, Friend RH, et al. Precursor route chemistry and electronic properties of poly( $p$-phenylenevinylene), poly[(2,5-dimethyl-p-phenylene)vinylene] and poly[(2,5-dimethoxy-p-phenylene)vinylene]. J Chem Soc Perkin Trans 1. 1992;1:32253231.

[36] O’Neill M, Kelly SM. Ordered materials for organic electronics and photonics. Adv Mater. 2011;23(5):566-584.

[37] Setia S, Sidiq S, De J, et al. Applications of liquid crystals in biosensing and organic lightemitting device: future aspects. Liq Cryst. 2016;43(13-15):2009-2050.

[38] Krikorian M, Voll CA, Yoon M, et al. Smectic A mesophases from luminescent sandic platinum(II) mesogens. Liq Cryst. 2016;43(12):1709-1713.

[39] Eccher J, Zajaczkowski W, Faria GC, et al. Thermal evaporation versus spin-coating: electrical performance in columnar liquid crystal OLEDs. ACS Appl Mater Interfaces. 2015;7(30):16374-16381.

[40] Wang Y, Cabry C, Xiao M, et al. Blue and green phosphorescent liquid-crystalline Iridium complexes with high hole mobility. Chem A Euro J. 2016;22(5):1618-1621.

[41] Aldred MP, Hudson R, Kitney SP, et al. Electroluminescent segmented liquid crystalline trimers. Liq Cryst. 2008;35(4):413-427.

[42] Billa MR, Kassireddy K, Haro M, et al. Liquid crystalline organic semiconductors: nematic spiro[cyclopentyl-1,9’]fluorenes. Liq Cryst. 2011;38:813-829.

[43] Gupta M, Pal SK. Triphenylene-based room-temperature discotic liquid crystals: a new class of blue-light-emitting materials with long-range columnar self-assembly. Langmuir. 2016;32(4):1120-1126.

[44] Bao WX, Billa MR, Kassireddy K, et al. Carbazole nematic liquid crystals. Liq Cryst. 2010;37:1289-1303.

[45] Jiang W, Li Y, Wang Z. Heteroarenes as high performance organic semiconductors. Chem Soc Rev. 2013;42:6113-6127.

[46] Shih PI, Chiang CL, Dixit AK, et al. Novel carbazole/fluorine hybrids: host materials for blue phosphorescent OLEDs. Org Lett. 2006;8(13):2799-2802.

[47] Brunner K, Dijken AV, Borner H, et al. Carbazole compounds as host materials for triplet emitters in organic light-emitting diodes: tuning the HOMO level without influencing the triplet energy in small molecules. J Am Chem Soc. 2004;126(19):6035-6042. 
[48] Zheng CJ, Ye J, Lo MF, et al. New ambipolar hosts based on carbazole and 4,5diazafluorene units for highly efficient blue phosphorescent OLEDs with low efficiency roll-off. Chem Mater. 2012;24(4):643-650.

[49] Kato S, Shimizu S, Kobayashi A, et al. Systematic structure-property investigations on a series of alternating carbazole-thiophene oligomers. J Org Chem. 2014;79(2):618-629.

[50] Wang X, Wang S, Ma Z, et al. Solution-processible 2,2'-dimethyl-biphenyl cored carbazole dendrimers as universal hosts for efficient blue, green, and red phosphorescent OLEDs. Adv Funct Mater. 2014;24(22):3413-3421.

[51] Liu C, Luo H, Shi G, et al. Luminescent network film deposited electrochemically from a carbazole functionalized AIE molecule and its application for OLEDs. J Mater Chem C. 2015;3:3752-3759.

[52] Cao Y, Yu G, Zhang C, et al. Polymer light-emitting diodes with polyethylene dioxythiophene-polystyrene sulfonate as the transparent anode. Synth Met. 1997;87(2):171-174.

[53] Tang H, Hessel CM, Wang J, et al. Two-dimensional carbon leading to new photoconversion processes. Chem Soc Rev. 2014;43:4281-4299.

[54] Rudati PS, Mueller DC, Meerholz K. The I-V characteristics of organic hole-only devices based on crosslinked hole-transport layer. J Appl Res Technol. 2015;13(2):253-260.

[55] Wang J, Xu X, Tian Y, et al. A novel blue fluorescent polymer for solution-processed fluorescent-phosphorescent hybrid WOLEDs. J Mater Chem C. 2015;3:2856-2864.

[56] Seymour RW, Cooper SL. Thermal analysis of polyurethane block polymers. Macromolecules. 1973;6(1):48-53.

[57] Menczel JD, Prime RB. Thermal Analysis of Polymers: Fundamentals and Applications, Canada: John Wiley \& Sons, Inc; 2009.

[58] Huo Y, Lu J, Lu T, et al. Comparative studies on OLED performance of chloro and fluoro substituted Zn(II) 8-hydroxyquinolinates. New J Chem. 2015;39:333-341.

[59] Lee SY, Adachi C, Yasuda T. High-efficiency blue organic light-emitting diodes based on thermally activated delayed fluorescence from phenoxaphosphine and phenoxathiin derivatives. Adv Mater. 2016;28(23):4626-4631. 